\title{
Building an Information Infrastructure for Enterprise Applications
}

\author{
Laura Haas \\ IBM Silicon Valley Laboratory
}

In a modern enterprise, it is inevitable that different portions of the organization will use different systems to produce, store and search their critical data. Competition, evolving technology, mergers, acquisitions, and geographic distribution all contribute to this diversity. Only by combining these various systems can the enterprise realize the full value of the data they contain. Yet building new applications across these various information sources can be amazingly painful, forcing developers to discover what data is where, figure out what it means, and learn myriad different interfaces.

An information infrastructure to address this challenge must offer users access to any data in the enterprise and the means of reaching additional data external to the enterprise, from partners, suppliers, etc. The infrastructure should provide appropriate integration technologies to support, alone or in combination, important enterprise usage patterns. It should connect islands of data into a single virtual information source, so that application development is vastly simplified.

To motivate this vision, this talk explores several real-life enterprise application challenges and the information technology needed to support them. It presents currently available technology building blocks, and identifies some future challenges in building a strategic information infrastructure for enterprise applications. 